\section{Management of blinding disease: loss of immunity and superinfection}

BG Evans

\begin{abstract}
Globally the most important loss of immunity currently occurs with HIV disease. The effects of HIV on the eye, since the advent of highly active antiretroviral therapy, have been less in countries where such treatment is available but even in such situations ophthalmic zoster can occur at higher CD4 cell counts and can still cause problems. Other opportunistic infections such as CMV retinitis tend to occur at lower CD4 cell counts. However, globally treatment is not universally available in resource poor countries where it is most needed. A major impact of HIV in such situations is on premature mortality affecting the health care and education workforce, which indirectly has an impact on blinding disease. In addition, loss of family income due to illness or death of parents can affect nutritional status of remaining family members especially children as well as the direct effect of opportunistic infections in the eyes of those infected with HIV.

Eye (2005) 19, 1035-1036. doi:10.1038/sj.eye.6701982
\end{abstract}

Keywords: HIV; blinding disease; global HIV impact

\section{Global issues with regard to HIV}

The global epidemic continues to give great concern - of an estimated 38 million people (UNAIDS estimate 2004) living with HIV at the end of 2003, 25 million are in sub Saharan Africa. However, the spread of the HIV epidemic in India and China, the world's two most populous countries, continues to grow. In 2003 worldwide an estimated 4.8 million people acquired HIV of whom 3 million were in sub Saharan Africa. Within Europe HIV spread in Russia and some of the newly independent states in Eastern Europe has been fuelled by injecting drug use and by spread to heterosexual partners - intensified by a large syphilis epidemic.

Within western Europe, all countries have seen a considerable decrease in AIDS diagnoses as treatment with highly active antiretroviral therapy (HAART) in those already HIV diagnosed has caused progression to an AIDS defining illness, effectively to cease. However, some people develop resistance or find HAART difficult to tolerate because of side effects.

\section{Within the UK}

Within the UK the epidemic among men who have sex with men continues with no evidence of a reduction in HIV incidence and in the last 3 years a rise in new diagnoses to around 2000 a year. The African epidemic has impacted on the UK significantly in recent years with almost half of new diagnoses (around 3000 in 2003) now acquired in Africa, mostly in people who have migrated from Africa. Rates of HIV transmission due to injecting drug use have remained low in the UK. Small numbers of people (around 300 in 2003) have acquired HIV within the UK through heterosexual intercourse ${ }^{1}$.

\section{Prevention}

Prospects for prevention worldwide and in the UK are problematic. An effective vaccine is almost certainly a number of years away; microbicides are potentially useful but so far an effective, cheap microbicide is not available; treatment which lowers viral load and therefore could and should have reduced incidence has been effective in halting disease progression but has had limited, if any, effect on halting transmission; and behavioural change, possible in theory, has been achieved in a handful of countries, but is difficult to replicate in all countries and not easy to sustain. Circumcision, which probably has a protective effect is a
Communicable Disease Surveillance Centre, London, UK

Correspondence: BG Evans, Communicable Disease Surveillance Centre, 61 Colindale Ave, London, NW9 5EQ, UK Tel: +44 208200 6868; Fax: +442082007868.

E-mail: barry.evans@ hpa.org.uk

Received: 7 April 2005 Accepted: 2 May 2005 
measure which is not easy to introduce safely in resource poor countries except in childhood.

\section{$C M V$ and herpes are the main eye-related infections}

Historically cytomegalovirus and herpes zoster have been the important infections affecting the eye in HIVpositive people. CMV retinitis is an AIDS defining illness, and usually appears at low CD4 cell counts (median 20; interquartile range 10-50) and thus is seen much less frequently now that treatment stops progression to such low counts. However, the complications of herpes zoster can occur at much higher CD4 cell counts. Other opportunistic infections affecting the eye, visual pathways or visual cortex are cryptococcal infection, toxoplasmosis and candida infections. Cerebral lymphomas, TB meningitis and HIV encephalopathy can all affect the visual cortex or visual pathways.

\section{HAART side effects}

Although most current treatments seem to have little in the way of side effects on the eye, this is an issue which needs to be considered as new drugs and drug combinations are developed.

\section{Other effects}

Within sub-Saharan Africa, in particular (but also being seen in other resource poor countries) HIV/AIDS is having other less direct effects on eye problems. This can be seen in the loss of skilled workers (nurses, health care assistants, doctors, etc) whose jobs entail preventing eye disease and diagnosing and caring for those with potential blinding disorders. Skilled workers are not easy to replace and in education and health care significant numbers of such workers are being lost to HIV/AIDS. In addition, many children have been orphaned and are being brought up by grandparents or the oldest sibling. The decrease in family income with the loss of both parents has an effect on nutritional status, ability to continue in education and other social effects which indirectly may affect eye disorders and blinding disease in such children.

\section{Conclusion}

In the past 21 years since HIV was discovered ${ }^{2}$ and the 23 years since the first case description of AIDS, ${ }^{3}$ this disease has had a very significant impact in many parts of the globe. Its interaction with tuberculosis has caused an upsurge in TB cases. Most of the HIV spread occurs in societies before effective preventive action is taken. In the third world many people die of other opportunistic infections before the opportunistic infections causing blinding disease occur. Within the west blinding disease due to opportunistic infections caused by HIV immunosuppression has been much less common since HAART.

\section{References}

1 The UK Collaborative Group for HIV and STI surveillance. Focus on Prevention. HIV and other Sexually Transmitted Infections in the United Kingdom in 2003. Health Protection Agency Centre for Infections: London, November 2004.

2 Montagnier L. Isolation of a T lymphotropic retrovirus from a patient at risk for acquired immune deficiency syndrome (AIDS). Science 1983; 200: 868-871.

3 Centers for Disease Control. Pneumocystis pneumonia Los Angeles. Morbid Mortal Weekly Rep 1981; 30: 250-252. 